\title{
Fear of Negative Evaluation and Perfectionism in Young Adults
}

\author{
GeetaSunkarapalli, TrishiAgarwal \\ (Department of Psychology, St. Francis College for Women, India) \\ (Department of Psychology, St. Francis College for Women, India)
}

\begin{abstract}
Fear of Negative Evaluation is defined as "apprehension about others' evaluations, distress over their negative evaluations, avoidance of evaluative situations, and the expectations that others would evaluate oneself negatively". "Perfectionism is a personality disposition characterized by striving for flawlessness and setting exceedingly high standards of performance accompanied by overly critical evaluations of one's behavior". The present study examined the relationship between fear of negative evaluation and perfectionism. The sample consisted of 200 young adults, divided into two (18-22 and 23-27 years). It is a quantitative study using purposive and snowball methods for sampling. The data was collected from the metropolitan city of Hyderabad, using Fear of Negative Evaluation scale and the Multidimensional Perfectionism Scale. IBM SPSS software, version 24 was used for the analysis of data using $t$-test and correlation. The results of the present study show a significant positive correlation between Fear of Negative Evaluation and Perfectionism, mainly socially prescribed perfectionism. The present study can be useful to develop an intervention for people with either high levels of fear of negative evaluation or socially prescribed perfectionism, as there exists a strong positive relationship between the two variables.
\end{abstract}

Keywords: Fear of Negative Evaluation, Perfectionism.

\section{Introduction}

According to Erikson (1959), when childhood and youth come to an end, life, so the saying goes, begins: by which we mean work or study for a specified career, sociability with the other sex, and in time, marriage and a family of one's own. According to his theory of psychosocial development, individuals between the ages 18 to 30 are in the stage of young adulthood (Feist\&Feist, 2008). A young adult's identity develops as a result of his or her perception and understanding of themselves in the context of social interactions. Developing strong interpersonal relationships and building a successful career become primary goals. Achieving one's goals requires them to aim for perfectionism in all the tasks and responsibilities they take on. This is successfully demonstrated by a number of studies conducted on perfectionism associating it with burnout (Ho et al., 2015) and stress (Dunkley et al., 2016). Perfectionism may be caused by certain factors like Fear of negative evaluation in an individual's life, which affect their motivation to succeed. Individuals who aspire to be perfect may be doing so to avoid Fear of negative evaluation in a social setting.

Fear of Negative Evaluation can be defined as "apprehension about others' evaluations, distress over their negative evaluations, avoidance of evaluative situations, and the expectations that others would evaluate oneself negatively" (Watson \& Friend, 1969). It can also be described as a fear that individuals experience when they find themselves in an evaluative situation. This fear, irrespective of their efficiency in performance, causes them to believe that others will evaluate them negatively or notice their shortcomings, which causes distress to the individual and leads them to avoid the situation altogether. They may also experience distress and discomfort in interpersonal communication.

Individuals with fear of negative evaluation may experience a sense of lack of social approval. Leary (1983) found that individuals tend to have more anxiety and try avoiding interpersonal contact when they have high levels of fear of negative evaluation. Among social evaluative situations, fear of negative evaluation has a stronger relationship with social anxiety than other anxieties like test anxiety or speaking anxiety (Howe, 2014). Talhat and Aslam (2012), found a positive correlation between psychological distresses, depression, anxiety and stress, and fear of negative evaluation. According to Kumar et al. (2015), there are no significant differences between men and women in the levels of fear of negative evaluation but there is a strong positive correlation between fear of negative evaluation and anxiety. Fear of negative evaluation is a marginal predictor of helping behavior. Participants with high scores on fear of negative evaluation are less likely to help. Individuals with low fear of negative evaluation are more inclined to help in non-social situations than individuals with a marginally higher fear (Karakashian et al., 2006).

Fear of negative evaluation causes differences in the way an individual processes information about himself and the way he processes information about others, in a social evaluative situation. An individual evaluates himself more negatively in a social evaluative situation than he would evaluate another individual. 
Fear of negative evaluation biases social evaluation learning. Fear of negative evaluation and learning negative social evaluation about self are positively correlated, meaning the more fear of negative evaluation an individual has the more he will evaluate himself negatively in social situations. Individuals with high levels of fear of negative evaluation are more sensitive to negative feedback or evaluation and individuals with comparatively low level of fear of negative evaluation disregard feedback and are insensitive to negative evaluations (Button et al., 2015). According to Levinson et al. (2013), there is a significant positive correlation between fear of negative evaluation and social appearance anxiety, social anxiety and maladaptive perfectionism. Fear of negative evaluation is significantly associated with socially prescribed perfectionism; a dimension of perfectionism (Flett et al., 1996).

"Perfectionism is a personality disposition characterized by striving for flawlessness and setting exceedingly high standards of performance accompanied by overly critical evaluations of one's behavior" (Frost et al., 1990; Hewitt \&Flett, 1991). Individuals, who strive for perfectionism, evaluate their behavior severely. They aim for flawlessness and do not accept any mistakes on their part. The three kinds of perfectionism are other-oriented perfectionism, self-oriented perfectionism and socially prescribed perfectionism (Hewitt et al., 1991). Individuals with Other-Oriented Perfectionism have very high expectations from others and want those expectations to be met. They set high standards for others and expect others to be perfect in their work. Otheroriented perfectionism is where an individual expects not himself but other people to be perfect. Individuals with Self-Oriented Perfectionism set very high standards for themselves. They expect themselves to be perfect; and harshly evaluate and criticize any kind of failure, mistake or flaw on their part. They strive to be perfect in everything they do. Individuals with Socially Prescribed Perfectionism strive to be perfect because their important others have very high expectations from them. They are always pressurized by the beliefs and standards set by others for them (Hewitt et al., 1991). Along with concern for meeting others standards, individuals with high levels of Socially Prescribed Perfectionism should also exhibit fear of negative evaluation (Hewitt \&Flett, 1991).

Socially prescribed perfectionism is significantly associated with procrastination; moreover, the link between procrastination and socially prescribed perfectionism was greater in men (Flett et al., 1992). There is an adaptive and maladaptive side to perfectionism as well. If the process of striving for excellence includes planning and organization of work, it is considered as adaptive perfectionism but if the process includes concern over failure and mistakes it may be maladaptive (Elison, \& Partridge, 2012). Adaptive and maladaptive perfection can coexist in an individual (Wigert et al., 2012). Perfectionism can also be divided into normal and neurotic. Individuals who take pride in their work and are high achievers are normal perfectionists as they allow themselves to be less accurate and make reasonable mistakes depending on the situation they are in. They are critical only to be exceptional. Individuals who are rarely satisfied with their performance, have no tolerance for mistakes and are overly critical of themselves are neurotic perfectionists as they are driven by a fear of failure and are more concerned with avoiding mistakes (Chang, 2014).Perfectionism is also related to self-conscious emotions like shame and guilt (Tangney, 2002), moral values, virtues and judgment (Yang et al., 2015), stress coping and burnout (Schwenke, 2012).

Keeping in mind the previous studies conducted on fear of negative evaluation and perfectionism, relationship between them can be assumed. Hence, the present study focuses on analyzing the relationship between the two variables.

\subsection{Research Questions}

(1) Is there a difference in the levels of Fear of Negative Evaluation and Perfectionism, between men and women in the ages $18-27$ years?

(2) Is there a difference in the levels of Fear of Negative Evaluation and Perfectionism, between the ages 18-22 and 23-27 years in young adults?

(3) Is there a relationship between Fear of Negative Evaluation and Perfectionism in young adults in the two age groups, i.e. 18-22 and 23-27 years?

\subsection{Objectives}

(1) To determine whether there are any significant differences in the levels of Fear of Negative Evaluation and Perfectionism and its three dimensions- Other Oriented Perfectionism, Self Oriented Perfectionism and Socially Prescribed Perfectionism, between:

a. men and women in the ages 18-27 years

b. men and women in the ages 18-22 years

c. men and women in the ages 23-27 years

d. the age groups 18-22 and 23-27 years old

e. the age groups 18-22 and 23-27 years old in women

f. the age groups 18-22 and 23-27 years old in men 
(2) To observe whether there is any relationship between Fear of Negative Evaluation and Perfectionism and its three dimensions- Other Oriented Perfectionism, Self Oriented Perfectionism and SociallyPrescribed Perfectionism:

a in men (18-27 years)

b in women (18-27 years)

c in the age group $18-22$ years

$\mathrm{d}$ in the age group 23-27 years

e in the total sample, i.e. 18-27 years olds

1.3 Hypotheses

A. There will be a difference in the levels of Fear of Negative Evaluation and Perfectionism and its three dimensions- Other Oriented Perfectionism, Self Oriented Perfectionism and Socially Prescribed Perfectionism, between:

(1) men and women in the ages $18-27$ years

(2) men and women in the ages 18-22 years

(3) men and women in the ages 23-27 years

(4) the age groups 18-22 and 23-27 years old

(5) the age groups $18-22$ and 23-27 years old in women

(6) the age groups 18-22 and 23-27 years old in men

B. There will be a relationship between Fear of Negative Evaluation and Perfectionism and its three dimensionsOther Oriented Perfectionism, Self Oriented Perfectionism and Socially Prescribed Perfectionism:

(1) in men (18-27 years)

(2) in women (18-27 years)

(3) in the age group 18-22 years

(4) in the age group 23-27 years

(5) in the total sample, i.e. 18-27 years olds

\subsection{Research Design}

II Method

The present study is a quantitative study in which Fear of Negative Evaluation is treated as the Independent Variable and Perfectionism with its dimensions Other Oriented Perfectionism, Self-Oriented Perfectionism and Socially Prescribed Perfectionism is treated as the Dependent Variable. This study adopts correlational and between groups design to determine whether there is any correlation between Fear of Negative Evaluation and Perfectionism in young adults.

\subsection{Sample}

The sample consisted of 200 young adults divided into two age groups- Group one included 100 individuals, 50 women and 50 men, between the ages 18 to 22 and Group two included 100 individuals, 50 women and 50 men, between the ages 23 to 27. Group one comprised of students and group two comprised of employed individuals. The mean age of the sample is 22.04 years. The mean age of women is 21.82 and of men is 22.15. The number of men is evenly distributed over the years in both the age groups. In the age group, 18-22 years, fifty percent of the women are 19 years old. In the age group, 23-27 years, almost fifty perfect of the women are 23 years old. Purposive and Snowball sampling techniques were used to collect the sample from the metropolitan city of Hyderabad.

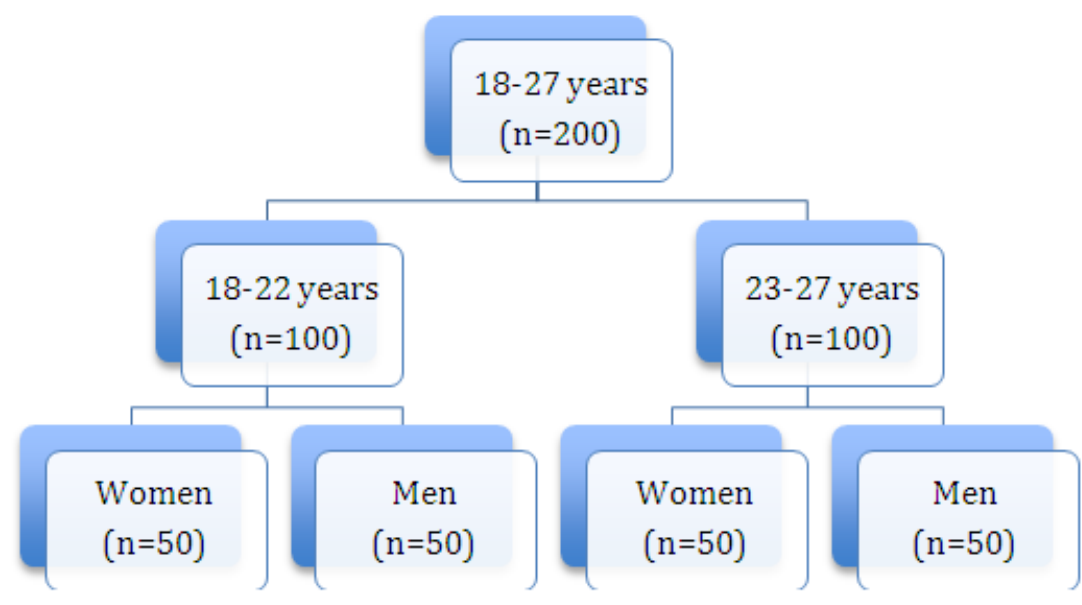


Figure 1 shows the division of the sample.

\subsubsection{Inclusion Criteria}

(1) Age of the participants: 18-27 years

(2) Qualifications of the participants:

- Participants aged 18-22 years: Students

- Participants aged 23-27 years: Employed

(3) Residing in different areas of Hyderabad

(4) Nationality of the participants: Indians

(5) Literate with a knowledge of English

\subsubsection{Exclusion Criteria}

(1) College dropouts for the age group 18-22 years.

(2) Individuals who have taken a break (a year or two off) between their studies for the age group 18-22 years

(3) Part time employees

(4) Self-employed individuals for the age group 23-27 years

(5) Non-Residential Indians

(6) Participants with known Physical or Chronic Illness

1.3 Instruments

Two questionnaires were used in this research along with an Information sheet. They were:

\subsubsection{Information Sheet}

Participants were asked to provide their age, gender, participation in prior research, nationality, employment status, education, the physical and psychological health of the respondents, and the like in writing, on the Information Sheet.

\subsubsection{Fear of Negative Evaluation Scale by David Watson and Ronald Friend (1969)}

The 30-item, self-rated scale measures discomfort and distress in interpersonal interactions. It is used to measure apprehension in individuals when they are negatively evaluated. The scoring key indicates whether or not to assign a value for yes or no answers. The final score, ranging from 0 to 30 , is the sum of all values. High scores indicate higher levels of fear of negative evaluation. The Cronbach alpha rating for the internal reliability is reported at 0.94 to 0.98 . The test-retest reliability was 0.78 to 0.94 according to Watson and Friend. Internal consistency of the Fear of Negative Evaluation scale was determined by correlating each item with the total Fear of Negative Evaluation score. The average to total score correlation was 0.72 .

\subsubsection{Multidimensional Perfectionism Scale by Hewitt and Flett $(1991,2004)$}

The 45-item scale includes three subscales- Other Oriented Perfectionism, Self-OrientedPerfectionism, and Socially Prescribed Perfectionism. It is a seven-point Likert-type scale ranging from Strongly Disagree (1) to Strongly Agree (7) designed to measure the level of perfectionism. Higher scores are indicative of greater levels of perfectionism in each subscale. Internal consistency of the three subscales was adequate, with Cronbach alphas of 0.86 for Self-Oriented Perfectionism, 0.87 for Socially Prescribed Perfectionism, and 0.82 for Other Oriented Perfectionism. The test-retest reliability of the MPS subscales is 0.88 for Self-Oriented Perfectionism, 0.75 for Socially Prescribed Perfectionism, and 0.85 for Other Oriented Perfectionism.

\subsection{Procedure}

After selecting the measures, a few arrangements were made for data collection. The questionnaires and the Information Sheet were prepared and organized. The researcher identified the individuals who fit the age group. Rapport was established with them and they were made aware that their participation in the study was purely voluntary. They were assured of maintaining confidentiality throughout the study. The individuals who agreed to participate in the study were requested to sign an 'Informed Consent Form'.

Next, the Information Sheet was administered. Based on the information schedule, the participants who fit the sampling criteria were selected. After the appropriate participants were screened out, instructions for the questionnaires (namely, the Fear of Negative Evaluation Scale and the Multidimensional Perfectionism Scale) were given first and the participants were requested to respond to the items. There was no fixed time limit for any of the questionnaires. However, the respondents completed the questionnaires in about 20 minutes.

\subsection{Statistical Analysis of Data}

Means and Standard Deviations of Fear of Negative Evaluation and Perfectionism along with its three dimensions were calculated for the total sample, for the age group 18-22 years, for the age group 23-27 years, for women and for men.t- Test was conducted for testing the significant difference for the categorizing 
variables, age and gender. Pearson's Product Moment Correlation was computed to determine whether any significant correlation exists between Fear of Negative Evaluation and Perfectionism and its three dimensions.

The sample mean for

\section{Results}

- Fear of Negative Evaluation is 11.66.

- Total Perfectionism is 190.53 .

- $\quad$ Self Oriented Perfectionism is 72.97.

- Other Oriented Perfectionism is 57.61.

- Socially Prescribed Perfectionism is 59.95.

Table 1:showing mean, standard deviation and $t$ values for Fear of Negative Evaluation (FNE) and Perfectionism (P) and its dimensions, Self Oriented Perfectionism (SOP), Other Oriented Perfectionism (OOP) and Socially Prescribed Perfectionism.

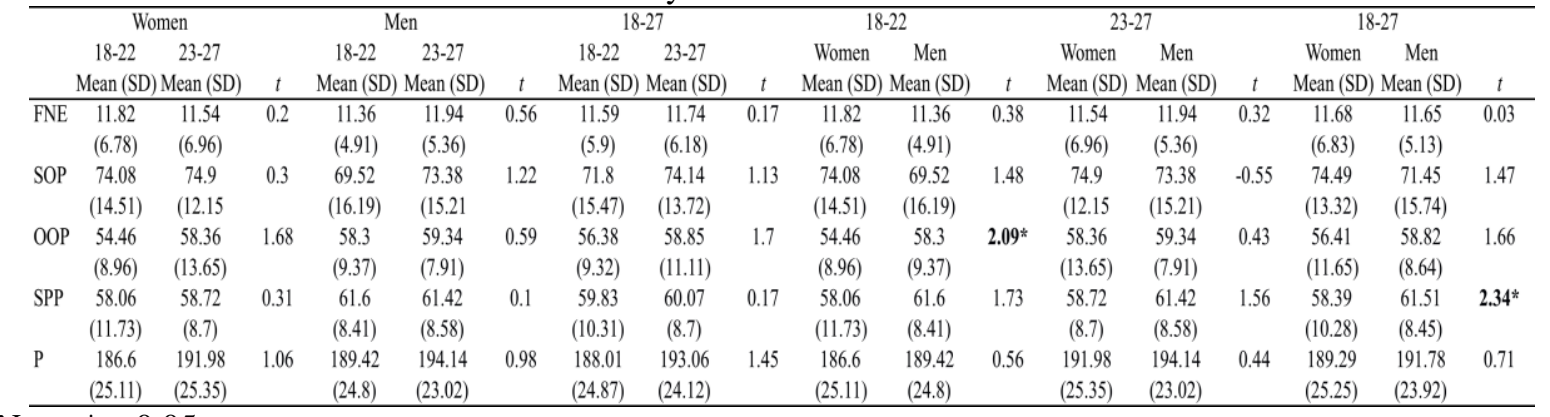

Note: $* \mathrm{p}<0.05$

$$
* * \mathrm{p}<0.01
$$

Table 1 shows a significant difference in the levels of Socially Prescribed Perfectionism, between women and men, in the total sample (18-27 years).There is a significant difference in the levels of Other Oriented Perfectionism, between women and men in the age group 18-22 years.

However, there areno significant differences in the levels of Fear of Negative Evaluation and Perfectionism and its dimensions (Self Oriented Perfectionism, Other Oriented Perfectionism and Socially Prescribed Perfectionism), between the age groups, 18-22 years and 23-27 years and in the total sample. There is no significant difference in the levels of Fear of Negative Evaluation and Perfectionism and its dimensions (Self Oriented Perfectionism and Socially Prescribed Perfectionism), between women and men in the age group 18-22 years.There is also no significant difference in the levels of Fear of Negative Evaluation and Perfectionism and its dimensions (Self Oriented Perfectionism and Socially Prescribed Perfectionism), between women and men in the age group 23-27 years.There is no significant difference in the levels of Fear of Negative Evaluation and Perfectionism and its dimensions (Self Oriented Perfectionism, Other Oriented Perfectionism and Socially Prescribed Perfectionism), between the age groups, 18-22 years and 23-27 years, in women.It also shows no significant difference in the levels of Fear of Negative Evaluation and Perfectionism and its dimensions (Self Oriented Perfectionism, Other Oriented Perfectionism and Socially Prescribed Perfectionism), between the age groups, 18-22 years and 23-27 years, in men.

Table 2:showing correlation between Fear of Negative Evaluation (FNE) and Perfectionism (P) and its dimensions, Self Oriented Perfectionism (SOP), Other Oriented Perfectionism (OOP) and Socially Prescribed Perfectionism.

\begin{tabular}{lccccc}
\hline & & & FNE & \\
& Women & Men & $18-22$ & $23-27$ & Total \\
\hline SOP & 0.05 & -0.18 & 0 & -0.1 & -0.05 \\
OOP & $\mathbf{0 . 2}^{*}$ & 0.01 & 0.02 & $\mathbf{0 . 2 2} *$ & 0.13 \\
SPP & $\mathbf{0 . 3 2}^{* *}$ & 0.04 & $\mathbf{0 . 2} *$ & $\mathbf{0 . 2 3} *$ & $\mathbf{0 . 2 1}{ }^{* *}$ \\
P & $\mathbf{0 . 2 5 * *}$ & -0.1 & 0.08 & 0.12 & 0.1 \\
\hline
\end{tabular}

Note: $* \mathrm{p}<0.05$

$* * \mathrm{p}<0.01$ 
Table 2 shows significant positive correlations between Fear of Negative Evaluation and Other Oriented Perfectionism, in the age group 23-27 years and in women.Socially Prescribed Perfectionism is positively correlated with Fear of Negative Evaluation in women, in the age groups 18-22, 23-27 and also in the total sample. Perfectionism is positively correlated with Fear of Negative Evaluation in women.

However there is no correlation between Fear of Negative Evaluation and Other Oriented Perfectionism, in the age group 18-22 years and in men. There is no correlation between Fear of Negative Evaluation and total Perfectionism along with its dimension Socially Prescribed Perfectionism, in men. There is no significant correlation between Fear of Negative Evaluation and total Perfectionism and its dimensions, namely, Self Oriented Perfectionism and Other Oriented Perfectionism, in the total sample.There is no significant correlation between Fear of Negative Evaluation and Self Oriented Perfectionism, in the age groups 18-22 years and 23-27 years and in women and men.

\section{Discussion}

The present study sought to find the relationship between fear of negative evaluation and dimensions of perfectionism. It also aims to find if there is any difference between the age groups and between men and women with respect to fear of negative evaluation and perfectionism.

Although there is no significant gender difference in the levels of Socially Prescribed Perfectionism in the subgroups, a significant gender difference is seen in the total sample. The mean score of Socially Prescribed Perfectionism is higher for men than for women, which would mean that men aspire for perfectionism more than women. This may be because the society, especially the Indian society, exerts more pressure on men to be successfulas they are considered the primary care takers of the family. Hence they are expected to be financially stable and secure. As men succumb to the pressure they tend to have higher socially prescribed perfectionism.

There is a significant gender difference in the levels of Other Oriented Perfectionismin the age group 18-22 years. Comparison of the means reveals that men have a higher level of Other Oriented Perfectionism than women, which means that men are more likely to expect perfectionism from others. Hewitt and Flett (1991) also found a significant gender difference in the levels of Other Oriented Perfectionism.

Significant differences were not found in the levels of Fear of Negative Evaluation between the age groups, 18-22 years and 23-27 years and also between men and women, in the total sample. No statistically significant gender difference has been found in the levels of Fear of Negative Evaluation (Yokus, 2013). This could be because today, women are at par with men in every sphere of life. They face similar challenges, socially evaluative situationsand hardships as men.However comparison of means reveals that women have higher levels of Fear of Negative Evaluation. In a study conducted by Karabulut et al. (2013) no statistically significant gender difference was found in the levels of Fear of Negative Evaluation, however after analyzing the mean scores it was found that women have a higher fear of negative evaluation when compared to men. West and Newman (2007) pointed out that during adolescence, parents and the society prevent girls from acting and behaving in a certain manner due to which they experience a higher level of social phobia. As a result the society expects girls to be calm and modest and in contrast expects boys to be more aggressive and assertive. Kumar et al. (2015) also found no significant gender differences in the levels of fear of negative evaluation however women scored slightly higher than men indicating that women have slightly higher fear of being rejected, judged or evaluated negatively. This could be because of the discrimination girls face in the society and the over-protectiveness of girls by parents. Society and family expect girls to possess certain qualities which may make girls hyper vigilant and sensitive to negative evaluation.

There is a significant positive correlation between Fear of Negative Evaluation and Socially Prescribed Perfectionism in the total sample. It is positively correlated inboth theage groups i.e., 18-22 years and 23-27 years. In a previous study conducted by Flett et al. (1996) it was found that higher levels of Fear of negative Evaluation was associated with Socially Prescribed Perfectionism. This could be because both these phenomena are altered due to the presence of other individuals. Individuals with Socially Prescribed Perfectionism are pressured by the beliefs and standards set by others for them. This pressure may arise due to fear of negative evaluation by others. Flett et al., (1996) also suggested that individuals with higher levels of Socially Prescribed Perfectionism adopt or develop a pattern ofwithdrawing and isolating themselves as a response or reaction to anticipated criticism from other individuals.

There is no significant correlation between Fear of Negative Evaluation and Other Oriented Perfectionism in the total sample that is in men and women aged between 18-27 years. However, there is a significant positive correlation between Fear of Negative Evaluation and Other Oriented Perfectionism in age group 23-27 years. This could also be because when an individual expects others to be perfect, he also believes that others expect him to be perfect. In order to achieve perfection, as suggested by Clark \& Wells (1995), an individual may evaluate oneself negatively and fear that others would also do the same.

There is a significant positive correlation between fear of negative evaluation and perfectionism, and it's dimensions other oriented and socially prescribed perfectionism in women. While explaining the traditional 
gender roles in the Indian society, Patel \&Parmentier(2005), suggest that emotional, supportive, nurturing, and submissive are considered as the feminine traits. A society, especially in India, has a specific set of norms for how a woman is supposed to behave. Young women find it difficult to subscribe to the gender roles as outlined by the society. They experience a constant fear of being evaluated if they fail to fit into the gender roles prescribed by the society. Hence they feel the need to be perfect.

\section{Conclusion}

The main finding of the study is that there is a significant positive correlation between Fear of Negative Evaluation and Perfectionism, mainly socially prescribed perfectionism.It was also seen that the level of Self Oriented Perfectionism is higher in the sample compared to the other two dimensions.

\subsection{Implications}

The finding of the present study can be useful to develop an intervention for people with either high levels of fear of negative evaluation or socially prescribed perfectionism, as there exists a strong positive relationship between the variables. Fear of Negative Evaluation and Socially Prescribed Perfectionism are both related to others' beliefs. Socially prescribed perfectionism posses societal stress and pressure on the individual which causes them to be perfect. Interventions can be devised to reduce the levels of fear of negative evaluation, which will change perfectionism from being socially prescribed to self-oriented. If perfectionism transforms to be self-oriented, the individual will be free from the stress and pressure and more productive and content.

\subsection{Limitations and Future Directions}

The present study was conducted in the metropolitan city of Hyderabad. A larger sample covering other cities of India could be taken. Cross-cultural studies could be conducted to understand the cultural differences with regards to the variables. The sample of the study included individuals from the age group 18-27 years. The study could be conducted on a different age group. A random sampling technique could be used to make generalizations.

\section{References}

[1] Erikson E. H. (1959), Identity and the Life Cycle: Selected Papers, Psychological Issues.

[2] Feist J., Feist G. J. (2008), Theories of Personality, McGraw-Hill Primis

[3] Ho, M. S., Appleton, P. R., Cumming, J., \&Duda, J. L. (2015). Examining the Relationship Between Perfectionism Dimensions and Burning Out Symptoms in Deaf and Hearing Athletes. Journal of Clinical Sport Psychology, 9(2), 156-172.

[4] Dunkley, D. M., Solomon-Krakus, S., \&Moroz, M. (2016). Personal standards and self-critical perfectionism and distress: Stress, coping, and perceived social support as mediators and moderators. In Perfectionism, health, and well-being (pp. 157-176). Springer International Publishing.

[5] Watson, D., \& Friend, R. (1969). Measurement of social-evaluative anxiety. Journal of consulting and clinical psychology, 33(4), 448 .

[6] Leary, M. R. (1983). A brief version of the Fear of Negative Evaluation Scale. Personality and Social Psychology Bulletin, 9(3), 371-375.

[7] Howe, J. (2014). Fear of Negative and Positive Evaluation across Social Evaluative Situations.

[8] Talhat, K., \&Aslam, N. (2012). Fear of Negative Evaluation and Psychological Distress among Patients of Drug Addiction. Journal of the Indian Academy of Applied Psychology, 38(3), 44-54

[9] Kumar, G., Athilakshmi, R., Maharishi, R., \& Maya, R. (2015). Relationship between Fear of Negative Evaluation and Anxiety.

[10] Karakashian, L. M., Walter, M. I., Christopher, A. N., \& Lucas, T. (2006). Fear of negative evaluation affects helping behavior: The bystander effect revisited. North American Journal of Psychology, 8(1), 13-32.

[11] Button, K. S., Kounali, D., Stapinski, L., Rapee, R. M., Lewis, G., \&Munafò, M. R. (2015). Fear of negative evaluation biases social evaluation inference: evidence from a probabilistic learning task. PloS one, 10(4), e0119456.

[12] Levinson, C. A., Rodebaugh, T. L., White, E. K., Menatti, A. R., Weeks, J. W., Iacovino, J. M., \& Warren, C. S. (2013). Social appearance anxiety, perfectionism, and fear of negative evaluation. Distinct or shared risk factors for social anxiety and eating disorders?.Appetite, 67, 125-133.

[13] Flett, G. L., Hewitt, P. L., \& De Rosa, T. (1996). Dimensions of perfectionism, psychosocial adjustment, and social skills. Personality and Individual Differences, 20(2), 143-150.

[14] Frost, R. O., Marten, P., Lahart, C., \&Rosenblate, R. (1990). The dimensions of perfectionism. Cognitive therapy and research, 14(5), 449-468.

[15] Hewitt, P. L., \&Flett, G. L. (1991). Perfectionism in the self and social contexts: Conceptualization, assessment, and association with psychopathology. Journal of personality and social psychology, 60(3), 456.

[16] Hewitt, P. L., Flett, G. L., Turnbull-Donovan, W., \&Mikail, S. F. (1991). The Multidimensional Perfectionism Scale: Reliability, validity, and psychometric properties in psychiatric samples. Psychological Assessment: A Journal of Consulting and Clinical Psychology, 3(3), 464.

[17] Flett, G. L., Blankstein, K. R., Hewitt, P. L., \&Koledin, S. (1992). Components of perfectionism and procrastination in college students. Social Behavior and Personality: an international journal, 20(2), 85-94.

[18] Elison, J., \& Partridge, J. A. (2012). Relationships between shame-coping, fear of failure, and perfectionism in college athletes. Journal of sport behavior, 35(1), 19.

[19] Wigert, B., Reiter-Palmon, R., Kaufman, J. C., \& Silvia, P. J. (2012). Perfectionism: The good, the bad, and the creative. Journal of Research in Personality, 46(6), 775-779.

[20] Chang, H. K. (2014). Perfectionism, anxiety, and academic procrastination: the role of intrinsic and extrinsic motivation in college students.

[21] Tangney, J. P. (2002). Perfectionism and the self-conscious emotions: Shame, guilt, embarrassment, and pride. 
[22] Yang, H., Stoeber, J., \& Wang, Y. (2015). Moral perfectionism and moral values, virtues, and judgments: A preliminary investigation. Personality and individual differences, 75, 229-233.

[23] Schwenke, T. J. (2012). The relationships between perfectionism, stress, coping resources, and burnout among sign language interpreters.

[24] Hewitt, P. L. \&Flett, G. L. (2004). Multidimensional Perfectionism Scale: Technical Manual. Toronto, Ontario: MultihealthSystems Inc.

[25] Yokus, H. (2013). Investigation of relationship between the Fear of Negative Evaluation and Musical Instrument Achievements of music teacher candidates (Gaziosmanpasa University Sampling). International Journal of New Trends in Arts, Sports \& Science Education (IJTASE), 2(3).

[26] Karabulut, E. O., Bahadir, Z., Certel, Z., \&Pulur, A. (2013, June). Investigation of fears of negative evaluation of young national kick boxers in terms of some variables. In Ovidius University Annals, Physical Education and Sport/Science, Movement and Health Series (Vol. 13, No. 2 Suppl., pp. 183-187). Ovidius University Press.

[27] West, Ae., Newman, D1., 2007, Childhood behavioral inhibition and the experience of social anxiety in American Indian adolescents (2007), Cultural Diversity and Ethnic Minority Psychology, 13 (3), 197-206.

[28] Clark DM, Wells A 1995. A cognitive model of social phobia. In: RG Heimberg, MR Liebowitz, D Hope et al. (Eds.): Social Phobia-Diagnosis, Assessment, and Treatment. New York: Guilford, pp. 69-93.

[29] Patel, R., \&Parmentier, M. J. C. (2005). The persistence of traditional gender roles in the information technology sector: A study of female engineers in India. Information Technologies \& International Development, 2(3), pp-29. 